\title{
IUFOST2006/1318 \\ Consumer Attitudes Towards Healthy Cereal Grain Products in Four European Countries
}

\author{
R. Shepherd
}

University of Surrey, Department of Psychology, University of Surrey, GU2 7XH Guildford, United Kingdom r.shepherd@surrey.ac.uk

\begin{abstract}
Whole grain products provide a number of potential heath benefits but in general these types of products are consumed to only a limited degree by consumers. In order to increase the consumption of both whole grain products and functional grain products it is necessary to understand the views of consumers about such products.

As part of the HEALTHGRAIN project (www.healthgrain.org) we have carried out a series of studies across four European countries (the UK, Finland, Germany and Italy). Four focus groups were carried out in each country (one with men and three with women). Overall the results from the four countries were similar with minor differences. For Finns rye was the most important grain and it was considered to be healthy and pleasant whilst rye products were relatively unknown in the UK and Italy. The Italians viewed normal pasta as being much healthier than did the other three countries but they were also very positive about the functional versions of this base product. Issues were raised about the believability of the different claims associated with various functional grain products. Whilst negative feelings were expressed with regards to genetic modification as a breeding process most people were positive about fortification and traditional breeding.

The focus groups were followed up with quantitative surveys involving around 500 consumers in each of the countries. This showed that although whole grain products were rated more positively than refined grain products in terms of healthiness, naturalness, being nutritionally balanced, filling and offering slow energy release, these effects were pronounced for the Finnish sample but the differences were much less for consumers from the UK and Italy. Women were found to be more aware of benefits of grain based foods than men, to be more health conscious and to expect less illness in later life caused by their eating habits. Men described themselves as paying less attention to a healthy diet but are conscious of possible health problems arising from this. Women were more willing to use grain products produced to have specific health benefits (e.g. bread containing added fibre). Older people were found to be more concerned about health and were also more willing to use a range of functional grain products than were younger consumers.

This study is financially supported by the European Commission in the Communities 6th Framework Programme, Project HEALTHGRAIN (FP6-514008).
\end{abstract}

